\title{
Number of Electroencephalogram Electrodes
}

National Cancer Institute

\section{Source}

National Cancer Institute. Number of Electroencephalogram Electrodes. NCI Thesaurus.

Code C154713.

The number of electroencephalogram electrodes used in the assessment. 